\title{
The Effect of 3D Visual Simulator on Children's Visual Acuity - A Pilot Study Comparing Two Different Modalities
}

\author{
Takeshi Ide $^{*}, 1,2$, Mariko Ishikawa ${ }^{3}$, Kazuo Tsubota $^{2}$ and Masaru Miyao ${ }^{4}$ \\ ${ }^{I}$ Minamiaoyama Eye Clinic, Tokyo, Japan \\ ${ }^{2}$ Department of Ophthalmology, Keio University, Tokyo, Japan \\ ${ }^{3}$ Denenchofu Eye Clinic, Tokyo, Japan \\ ${ }^{4}$ Graduate School of Information Science, Nagoya University, Nagoya, Japan
}

\begin{abstract}
Purpose: To evaluate the efficacy of two non-surgical interventions of vision improvement in children.
Methods: A prospective, randomized, pilot study to compare fogging method and the use of head mounted 3D display. Subjects were children, between 5 to 15 years old, with normal best corrected visual acuity (BCVA) and up to $-3 \mathrm{D}$ myopia. Subjects played a video game as near point work, and received one of the two methods of treatments. Measurements of uncorrected far visual acuity (UCVA), refraction with autorefractometer, and subjective accommodative amplitude were taken 3 times, at the baseline, after the near work, and after the treatment.

Results: Both methods applied after near work, improved UCVA. Head mounted 3D display group showed significant improvement in UCVA and resulted in better UCVA than baseline. Fogging group showed improvement in subjective accommodative amplitude. While 3D display group did not show change in the refraction, fogging group's myopic refraction showed significant increase indicating the eyes showed myopic change of eyes after near work and treatment.

Discussion: Despite our lack of clear knowledge in the mechanisms, both methods improved UCVA after the treatments. The improvement in UCVA was not correlated to measured refraction values.

Conclusion: UCVA after near work can be improved by repeating near and distant accommodation by fogging and 3D image viewing, although at the different degrees. Further investigation on mechanisms of improvements and their clinical significance are warranted.
\end{abstract}

Keywords: 3D, fogging , myopia, vision, visual acuity.

\section{INTRODUCTION}

Visual acuity is not constant throughout the life time. Human babies born at full term are usually born hyperopic as many other animals [1-3]. Macular immaturity at birth is also the limiting factor for visual acuity of the new born [4]. Axial elongation of the eye occurs during the development of the vision in early stage of life, and it is a regulated process known as emmetropisation $[5,6]$.

However, in some cases, this tendency to become myopic continues or re-starts to pose visual acuity issues. Severe myopia is highly prevalent especially among some ethnic groups [7], and studies suggest the presence of genetic influences [8-10]. Also, Taylor et al. suggested from the study on the Australian Aborigine children, that the dietary change and formal education may have contributed to myopic shift within past 20 years [11]. Similar reports from East Asia suggest more intensive educational demand to

*Address correspondence to this author at the Minamiaoyama Eye Clinic Tokyo. Renai Aoyama Building 4F 3-3-11 Kitaaoyama, Minato-ku, Tokyo 107-0061, Japan; Tel: +81-3-5772-1440; Fax: +81-5772-1442;

E-mail: teyede@minamiaoyama.or.jp children and increased viewing of electronic display as the factors for myopic shift $[12,13]$.

Thus, myopia is suggested to be a multi-faceted phenomenon, and variety of attempts for its treatment were made throughout the world, including medical therapies [14], lens and eye exercises [15,16], and massage therapies [17]. Unfortunately, these attempts did not achieve cure or became the base for standard treatment.

One of the mechanisms suggested as a trigger for myopia progression is the sustained accommodation spasm of ciliary muscles, which is caused when we do close-up tasks for a long time with uncorrected hyperopia, emmetropia, or corrected myopia. When the eye views the distance, the ciliary muscles are relaxed as seen in distance accommodation, but when viewing near, they are contracted, which is the near accommodation (Fig. 1).

To relieve this spasm, the fogging method [9] and the dilating drops like atropine have been widely used and it was reported to have substantial effects with non-accomodative mechanism as observed in chick-eye experiment reported by McBrien et al. $[18,19]$. Others included electrostimulation [19] and multifocal contact lens [20-25]. In addition, 3D 

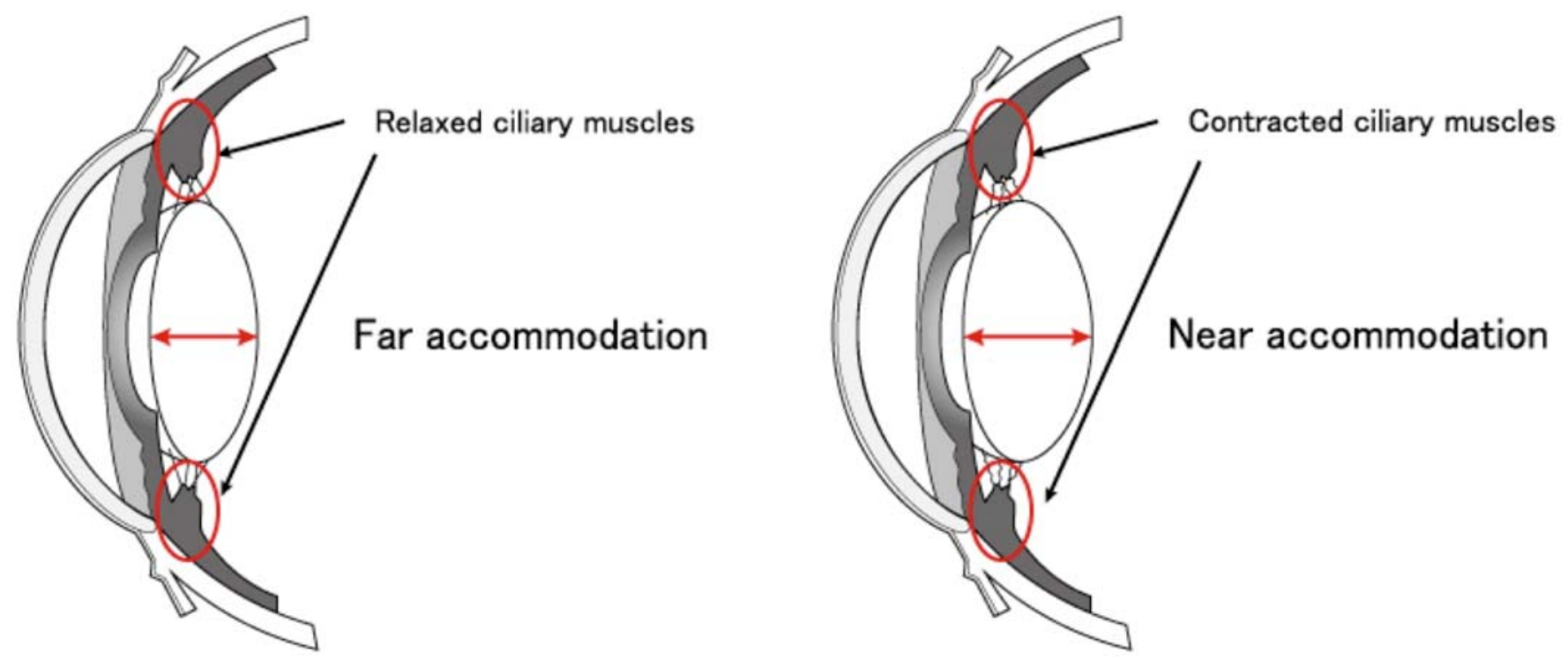

Fig. (1). During the far accommodation, ciliary muscles relax, and the crystalline lens thickness decreases. On the other hand, ciliary muscles contract during near accommodation resulting in thicker lens.

image stimulator which was approved as a medical device has been used in our clinics for purposes such as relaxing the over-tensed muscle $[26,27]$. In our clinical impression, these two modalities work, but we have not reported the short-term and long-term effect on the UCVA.

In this pilot study, we aimed to compare the short-term effects of these two modalities (fogging and 3D image) for the purpose of developing the effective care for myopia in the future. Our objective is to determine the effects of two methods on visual acuity after near work burden. We believe this is a rare original study on pediatric subjects, since some studies were conducted with youth and adults $[28,29]$.

\section{MATERIALS AND METHODS}

We performed a prospective randomized study comparing two methods of non-surgical intervention. Planned enrollment was 25 children. Subject inclusion criteria were healthy children, between 5 to 15 years old, with BCVA of 20/20 and within -3D of myopia. The children and their parents or legal guardians must be willing to comply with the instructions during the treatment, able to come to clinic for the study, and come to the follow-up visits. Exclusion criteria include inability to follow instructions, and having active ocular diseases.

The procedures used conformed to the tenets of the Declaration of Helsinki, and an informed consent was obtained from both the patients and the parents or legal guardians after being given an explanation of the procedures to be used. The study was approved by Institutional Review Board (IRB).

Subjects were age-matched and randomly assigned to two groups: Group A, a binocular vision tester for fogging; Group B, head mounted 3D display. Measurements were taken at three time points: before the 30-minute near work, after the task, and after the treatment with one of the two modalities (Fig. 2).
Measured parameters were: UCVA, refraction with autorefractometer, and subjective accommodative amplitude using an existing subjective accommodometer, D'ACOMO (World Optical Corporation, Kyoto, Japan). D'ACOMO is a system designed to measure the accommodative power in as setting of natural gaze by fixation on a target. In the image display, the viewer sees the object moving from $50 \mathrm{~cm}$ to 5 $\mathrm{cm}$ at the speed of $0.2 \mathrm{D} / \mathrm{sec}$. For the evaluation of UCVA, we have selected Log MAR visual acuity, which is the logarithm of the minimal angle resolution. It is one of the most widely accepted scientific expressions of vision and better suited to the statistical analysis of the data. Log MAR VA of 0.0 is equivalent to 20/20 in common fraction system by foot unit and 1.0 in decimal system. Log MAR of 1.00 is 20/200 or 0.10 ; and, Log MAR of -0.1 is about $20 / 16$ or 1.20 [30]. As the Log MAR VA is smaller, vision is regarded better. The refraction determined by autorefractometer provides the information of the level of refraction. In this measurement system, the negative value such as $-10 \mathrm{D}$ (diopter) is myopia, emmetropia is 0 , and hyperopia is positive value. Spherical equivalent refraction is equal to the algebraic sum of the value of the sphere and half the value of the cylinder.

The near work is the video game played on handheld portable player for 30 minutes. The subjects played the same game program continuously as the near work, using complete correction by trial frame and lenses.

Treatments for two groups were as follows:

Group A, Fogging by D-5000 AUTO (World Optical Corporation, Kyoto, Japan), which is designed to relieve habitual near accommodation. The apparatus shows the 6 scenes of stereoscopic photos to the viewer, indirectly stimulating ciliary body muscle to relieve its tension [31]. This is called fogging and known to lessen eye strain and realign the line of vision parallel. The images let the patient eyes to track from near scenery to distance view, from $+3.00 \mathrm{D}$ (fogging) to $\pm 0.00 \mathrm{D}$ to $-1.00 \mathrm{D}$ (resting position of accommodation) during 5-minute treatment time. 


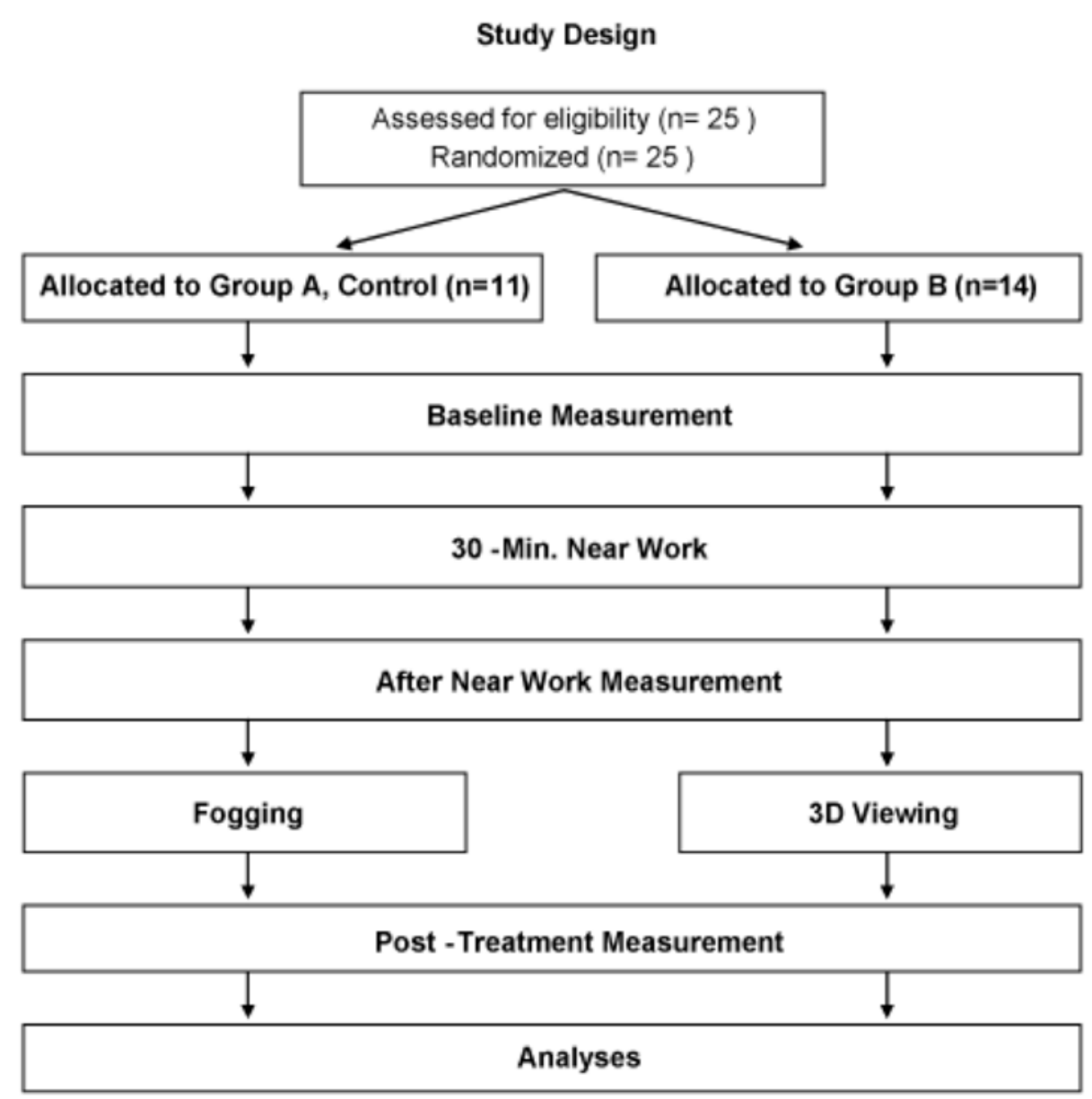

Fig. (2). The design of this study is a two-group randomized study, allocated subjects to either fogging or $3 \mathrm{D}$ viewing interventions.

\section{Group B, Head Mounted 3D Display}

Head mounted display system (HMD) Sky Crystal OLYMPUS POWER 3D (Olympus Visual Communications, Tokyo, Japan) was used (Fig. 3). The subjects wear the goggle part as eye glasses. The system shows a video of a spherical object in the center that appears far initially then close, again move away far (Fig. 4). The video is 10 seconds long and automatically repeats for 5-minute viewing time, which repeats distance accommodation and near accommodation, using stereoscopic image to let the patients eye track from $0.1 \mathrm{D}$ to $10 \mathrm{D}$.

\section{Statistical Analysis}

The data obtained from the subjects in two groups were analyzed for its change in measured parameters. To evaluate the statistical significance of the difference in the Log MAR visual acuity between the measurements one-way analysis of variance (ANOVA) was used with the Scheffé method for UCVA, between the baseline measurement and after the treatment, and after the near work and after the treatment. For the statistical analysis, we have selected Log MAR visual acuity. This is a liner scale, and one of the most frequently used visual acuity measurement with smaller number indicating better vision. The average of Log MAR visual acuity of right and left eyes of the each subject was used [32]. P value less than .05 was considered to indicate a significant difference.

\section{RESULTS}

Among all enrolled, the percentage of male was $72 \%$ (18 boys and 7 girls). In Group A, the percentage was $71.4 \%$ (10 boys and 4 girls), and in Group B, 72.7\% ( 8 boys and 3 girls). The average age of two groups were similar. Subjects had Log MAR UCVA ranging from -0.07 to 1.40 at the baseline. Average \pm SD for each groups were: $0.59 \pm 0.31$ for Group A and $0.71 \pm 0.36$ for Group B. There was no significant difference in the baseline UCVA between two groups ( $\mathrm{P}=0.146$, t-test, both sides).

Fig. (5a) shows the visual acuity at each stage and the difference between them during this experiment. Interestingly, even a short visual burden affected the visual acuity, as the measurements of mean Log MAR visual acuity of both groups increased indicating the decrease in vision. Both group showed improved vision after the treatment; however, Group B reported significant improvement of UCVA from baseline measurement before playing game of 0.71 to after the treatment of $0.64(P<0.05)$, whereas Group A did not.

Fig. (5b) shows accommodative amplitude at each stage. Group A showed significant increase after near work and treatment, indicating better vision for viewing closer objects. Group B reported no significant difference between the stages. 


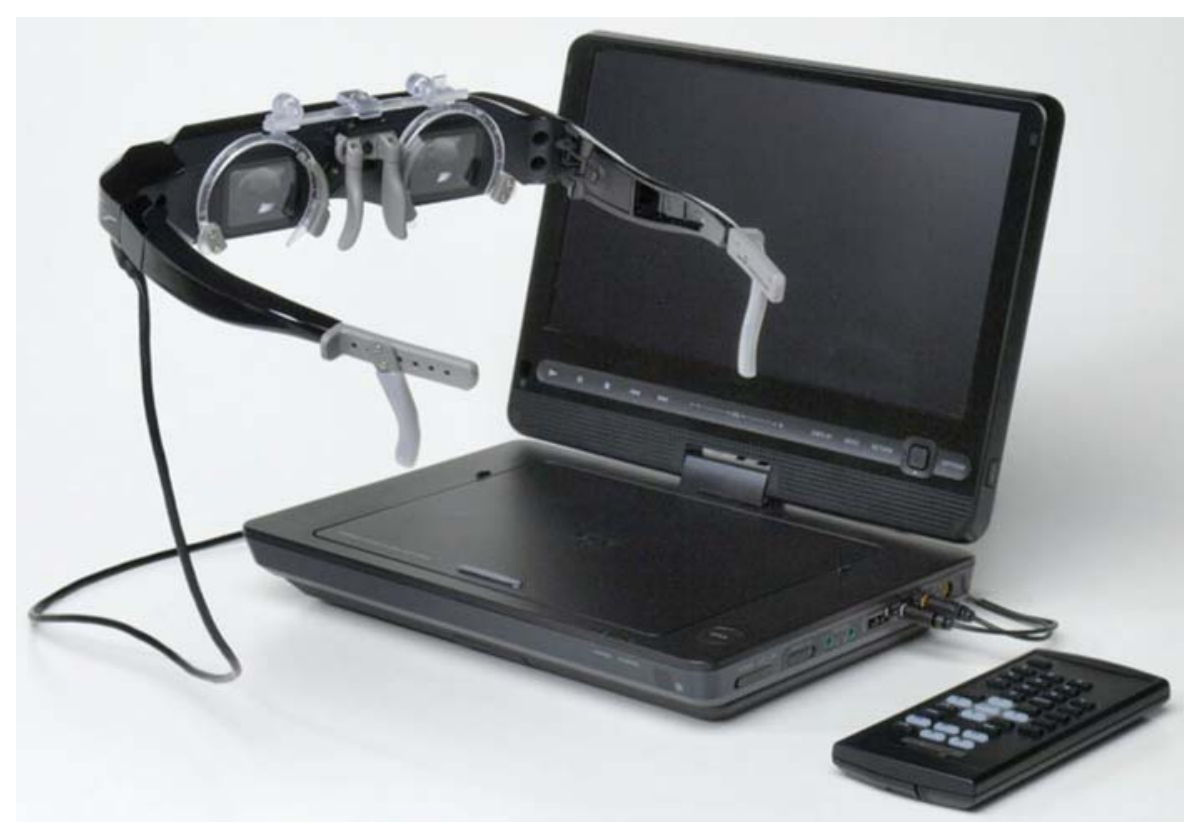

Fig. (3). Head mounted display system, Sky Crystal OLYMPUS POWER 3D. Manufactured by Olympus Visual Communications, Tokyo, Japan

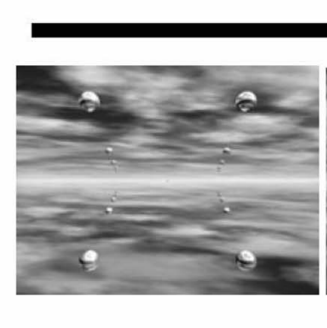

Far

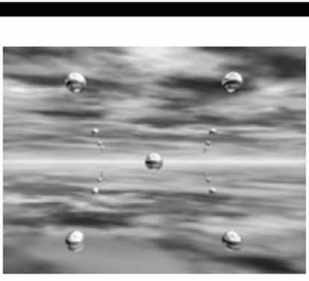

$\Leftrightarrow+\frac{e}{2+2}$

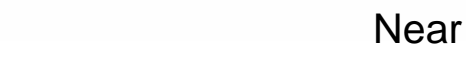

Near

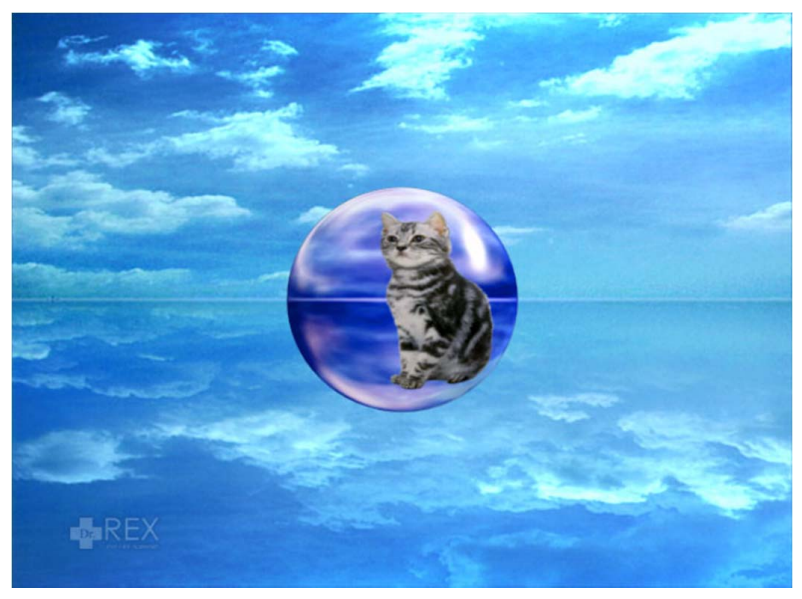

Fig. (4). Images used by head mounted display system.

Fig. (5c) shows the spherical equivalent refraction at each stage. Group A showed significant difference between baseline and after the treatment, but no significant difference was observed between baseline and after near work, and after near work and after treatment. Group B showed difference in refraction after near work; however, between the near work and the treatment or baseline and after the treatment did not show the significant difference.
In the next analysis, we compared the improvements between baseline and after treatments between two treatment groups (Fig. 6a-c). There was significant difference in UCVA improvement between Group A and Group B, with latter showing more improvement in visual acuity (Fig. 6a). Subjective accommodative amplitude result was similar in both groups (Fig. $\mathbf{6 b}$ ). 


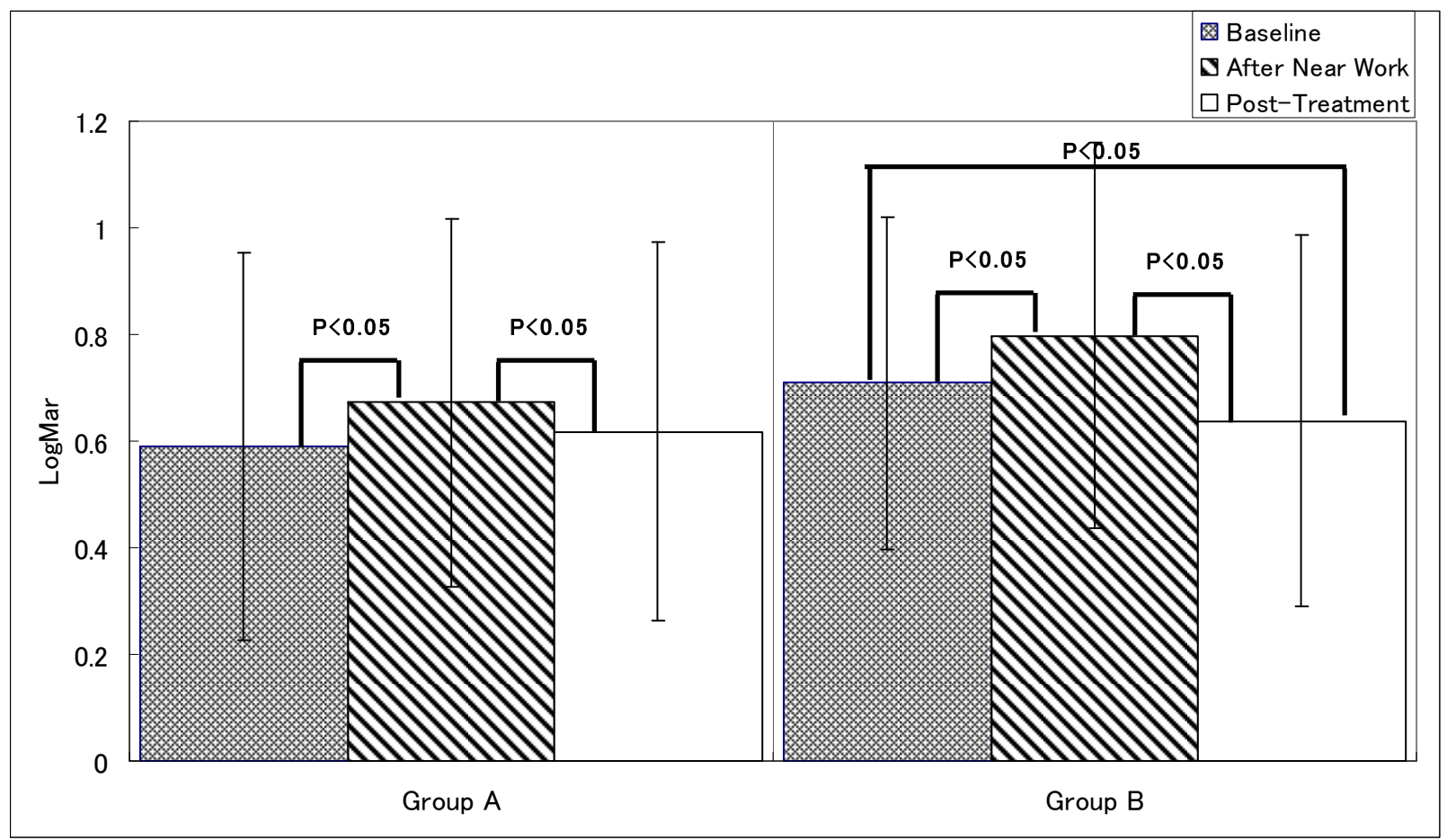

Fig. (5a). The mean Log MAR visual acuity measurements of both groups increased after visual burden, which means decreased vision, and patients' eyes became more myopic. Subsequent treatment by either method improved the UCVA, but at the different degree.

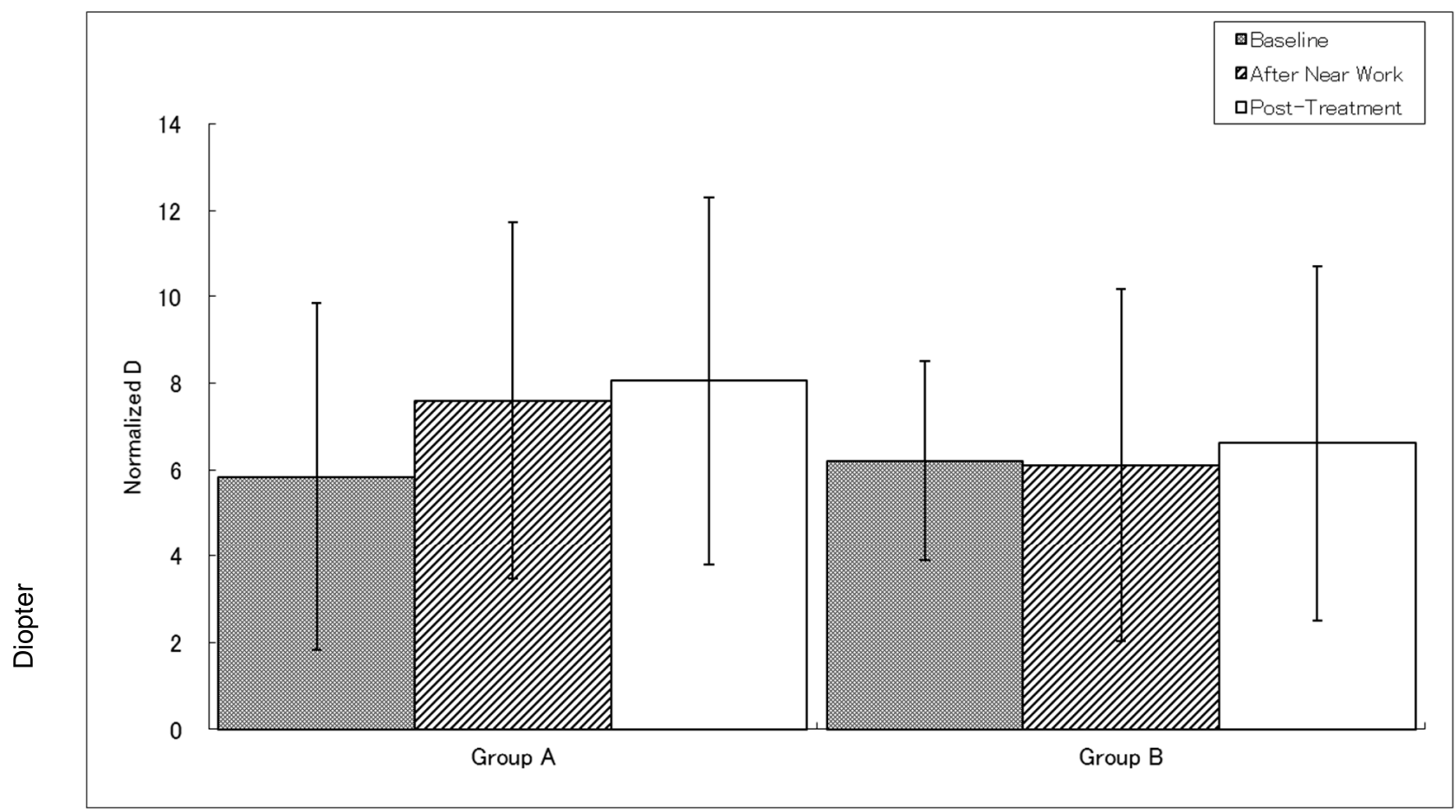

Fig. (5b). Near vision is the vision for objects $30 \mathrm{~cm}$ form the viewer. In this normalized Diopter measurement, larger number indicates better near visual acuity. Group A subjects showed increased near vision measurements indicating the eyes were accommodated to view near objects, or became myopic, even after the treatment. Note: Diopter is the inverse of focal distance, and this is commonly used to show the degree of myopia. Normal vision, emmetropia is $0 \mathrm{D}$, meaning the image is formed on the retina. However, low myopia, which is from 0 to $3 \mathrm{D}$ forms image before reaching retina, indicated by negative figure. Medium myopia is between $-3 \mathrm{D}$ to $-6 \mathrm{D}$, and high myopia is $-6 \mathrm{D}$ or less. In this graph the normalized value is used that is without negative sign. 


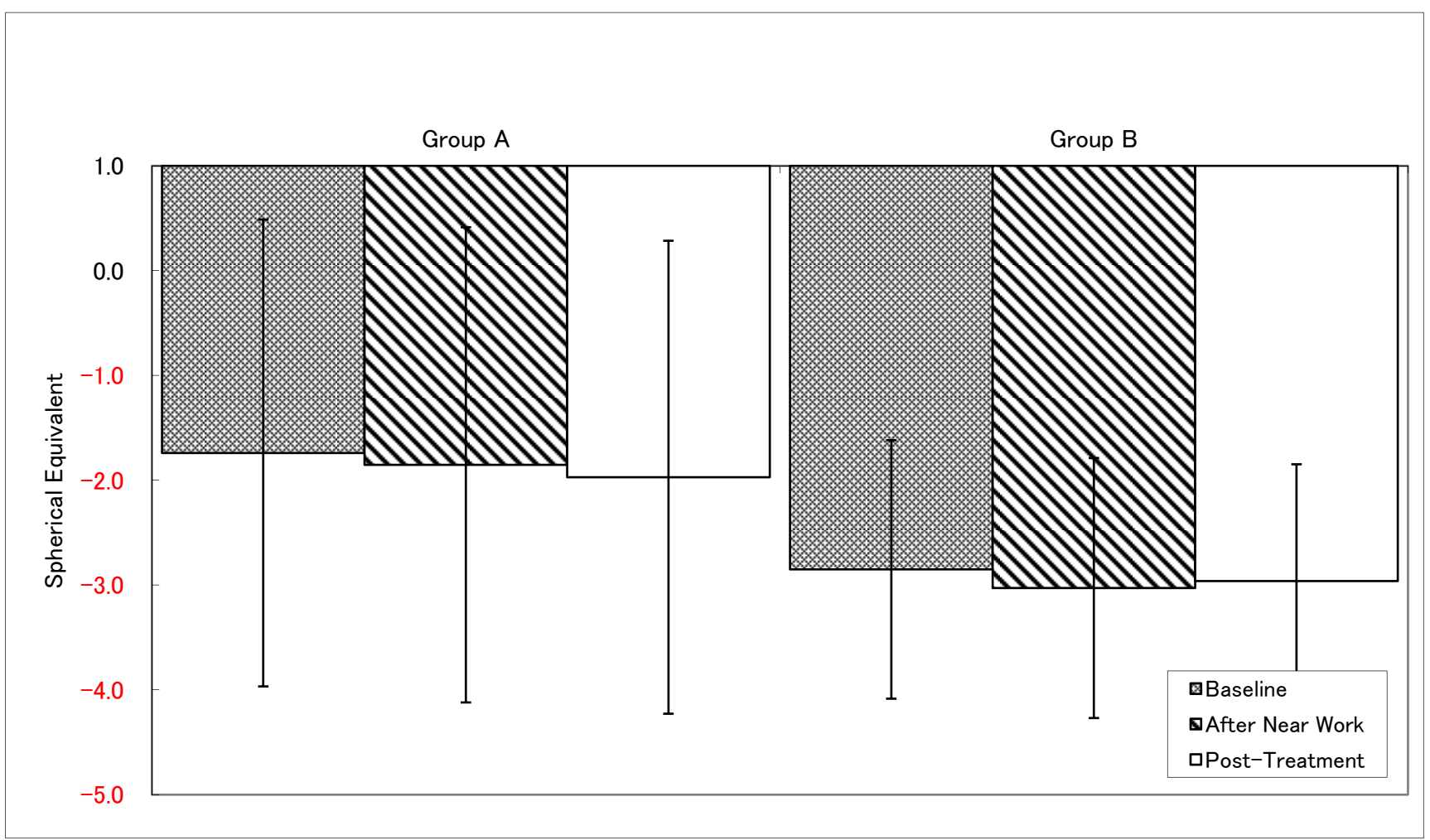

Fig. (5c). Decreasing value in spherical equivalent means myopic change. Although the change in refraction was small, Group A showed increased myopia. Group B did not show the difference between baseline and post-treatment despite the change in UCVA.

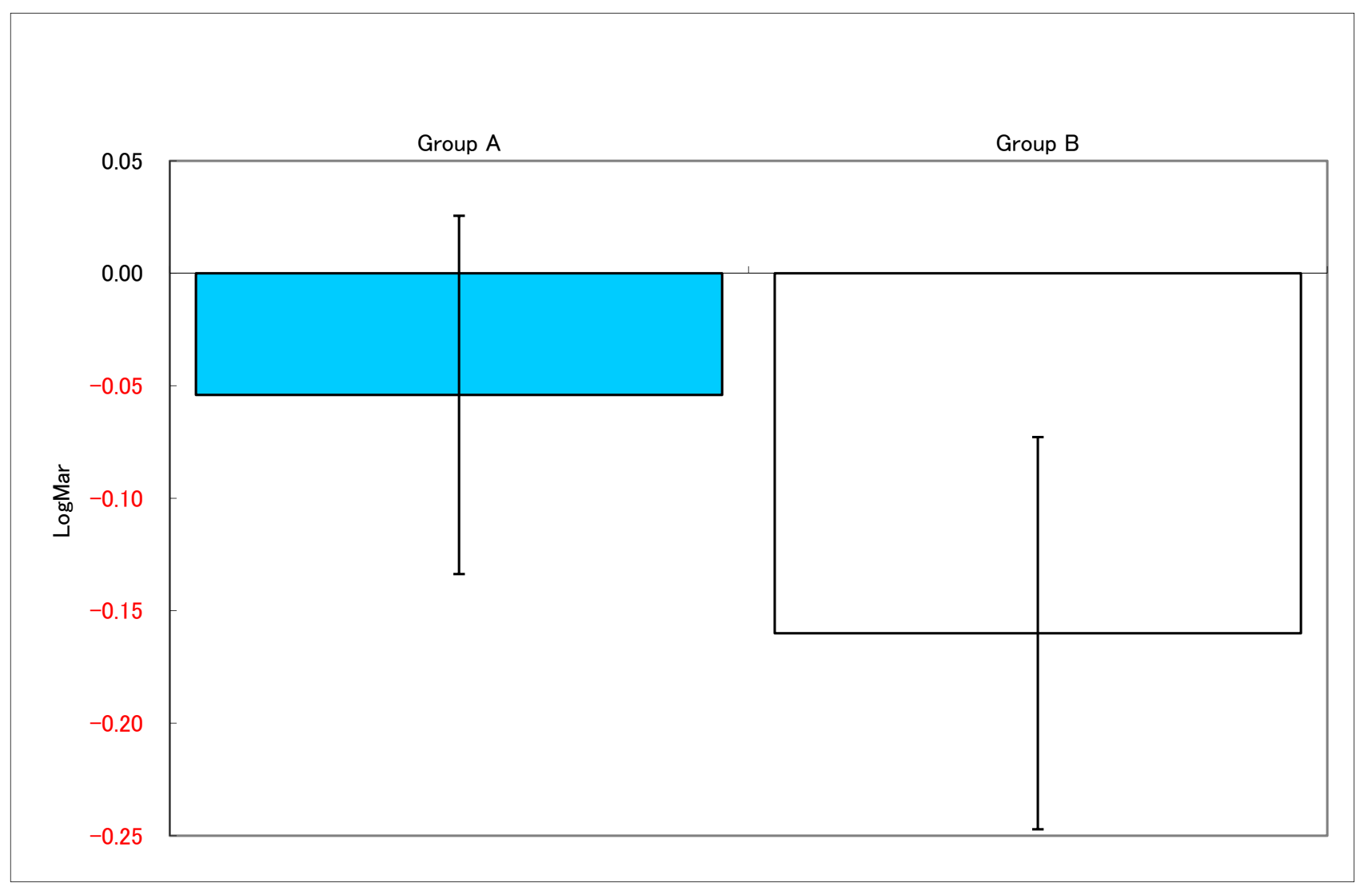

Fig. (6a). Group B had larger decrease in LogMAR visual acuity, meaning more improvement in UCVA. Significant difference was observed between two groups. 


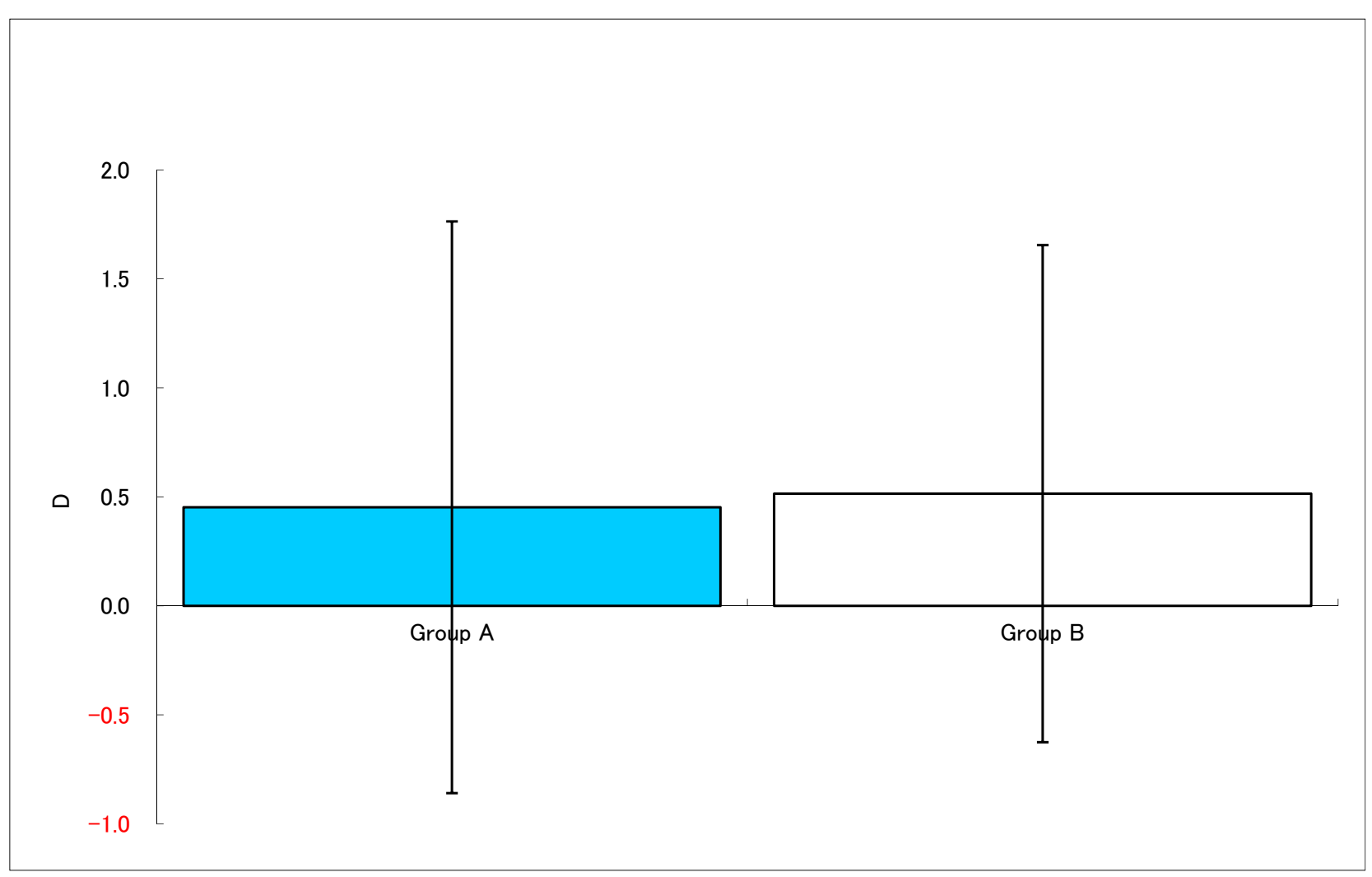

Fig. (6b). No substantial difference in near point measurements.

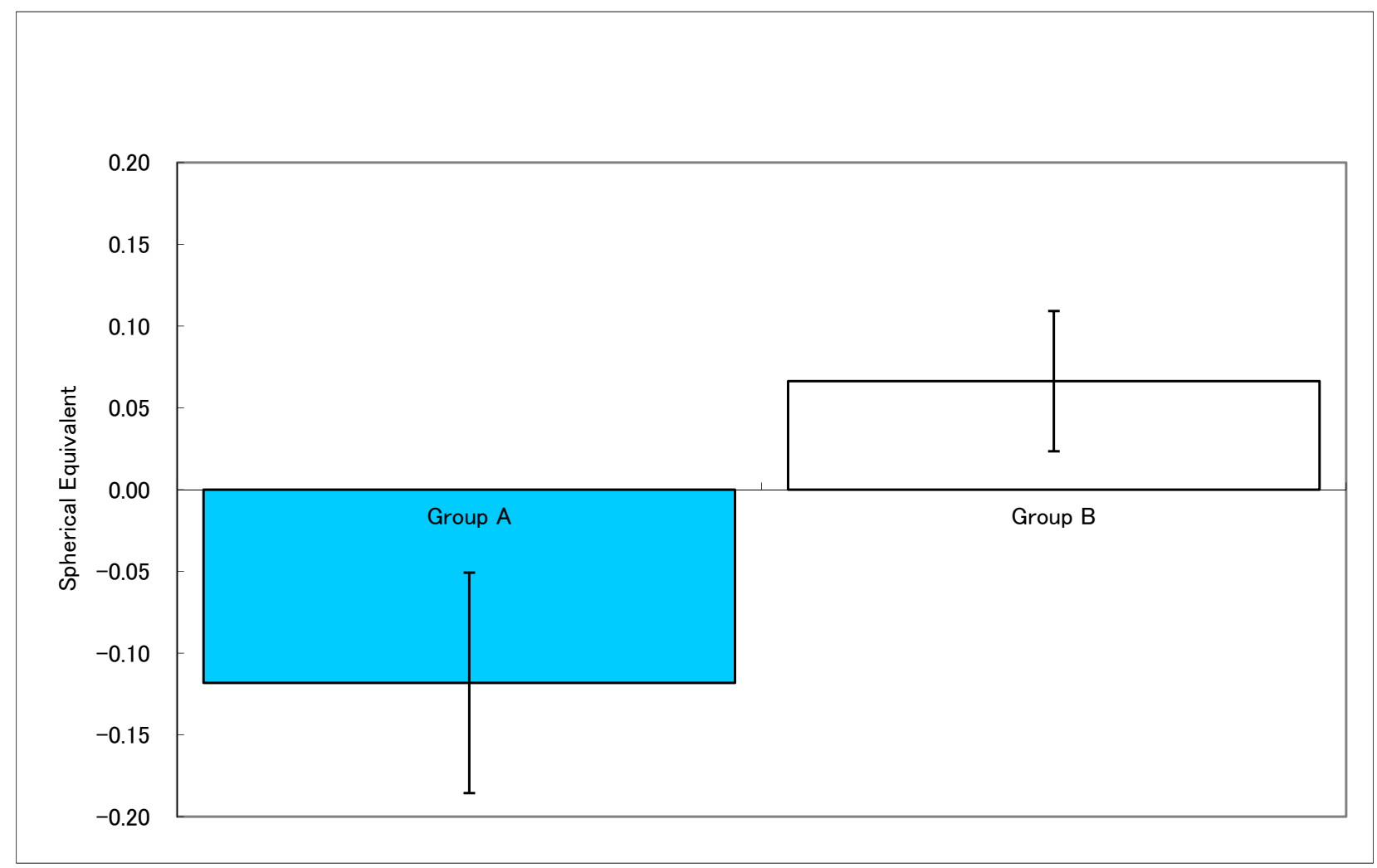

Fig. (6c). Refraction of the eyes changed after near work to post-treatment. The decrease in Group A shows the eyes were myopic. No significant change was observed in Group B. 


\section{DISCUSSION}

Myopia is the most common reason for visual impairment among the youth and it is a complex phenomenon. Increased scleral elasticity, genetics as seen in family history, reading, computer use, life style, diet, and more factors are cited as the possible causes of myopia. As one of the factors, hypertonic contraction of ciliary muscle to increase the lens thickness is cited as one of the probable reasons for this impaired accommodation [32-35].

Recently, a report on the evidence has suggested that central and peripheral defocus may have significant impact on the axial elongation associated with myopia development and progression [36]. In animal study model, progression of axial length is triggered with mechanical pressure on the chick eye by increased intraocular pressure [37,38]. On the other hand in human, there is an evidence suggesting the time spend outdoor may be protective and prevent myopia development $[39,40]$. Although this phenomenon may be species dependant, once this cascade of myopia progression is initiated, the vicious cycle starts and eyes experiences rapid progression of myopia; though, this is just one of the supposed mechanisms [8].

Our study became possible due to the advancement of technology that offers three-dimensional and moving images, traveling between near and far, without using massive apparatus required previously. Use of the projected image is even more practical as the software technology can provide different images easily for children to view as well as adults. With such technological background, we were able to select two different methods to compare their effects on visual acuity. Due to the novelty of the technology, we could not find reliable study investigating 3D image viewing and visual acuity or refraction.

D-5000 Auto is widely used in clinics throughout Japan for fogging, a treatment offered before examinations for eyeglass prescription to relieve habitual near accommodation. Originally, fogging is performed for the purpose of avoiding over-corrections of myopia or detecting latent hyperopia. Head-mounted 3D display used in Group B shows moving stereoscopic image by large sunglasses-like apparatus. Both were applicable even to children and we were able to perform this study and obtain data from subjects as young as 7 years old.

As a result of our study, both treatment modalities applied after the near work visual burden were effective to improve UCVA (Fig. 5a). We even observed significant improvement compared to the baseline and after the treatment by fogging and head mounted 3D display (Group B, Fig. 5a).

Fogging method did make significant changes in the UCVA after treatment, but not to the extent to improve the vision compared to the baseline (Fig. 5a). Our analysis comparing baseline and after treatment results showed similar subjective accommodative amplitude between the groups (Fig. 6b), but the refraction results showed hyperopic (myopic correction) tendencies by using 3D display (Fig. 6c). Furthermore, when determining accommodative amplitude, the same device, D'ACOMO is used so that there may be some additive effect to the eyes, meaning the patient may be showing the effect of additional treatment.
From these results, we see possibilities in using these methods to improve myopia in children and teenagers, and suggest further studies.

Repeating distant and near accommodation with stereoscopic image, the subject can stretch or contract the muscles involved in ciliary body and lens accommodation. We also observed the different rate of vision improvements and refraction improvements: In group A, vision improvement was observe in $36 \%$ of subjects, and refraction improvement in $14 \%$. In group B, $85 \%$ and $54 \%$, respectively. These data show that some eyes' vision improved without the change in refraction.

We observed in some cases with large degree of improvements in visual acuity without significant change in the refraction. The relaxation of ciliary muscle may be one of the factors behind observed improvement $[41,42]$. Therefore, the visual improvement may not be explained at refraction only.

However, as a limitation of this study, the results or any improvement observed here may not reflect the full magnitude of the treatment effects. The data for this study was taken only once, after single near work and single treatment, but both methods were designed to be used regularly aiming for the long term effects. Therefore, the level of improvements in the single treatment may not be sufficient to determine the long term effect. There was difference in average refraction at the baseline between groups. In addition, the patients enrolled in this study were the children and teen agers already visiting the clinic on regular bases, for exam and other treatments. Their results may differ from virgin eyes of children from general public. We need to take the measurement error into account, especially the refraction determined by autorefractometer. The instrument induced myopia could add myopic tendency to the results, especially in young children $[43,44]$.

Additionally, we need to mention that there are nonresponders to any method. From our experience of offering vision improvement treatment including electrostimulation, we have seen certain numbers of patients do not receive the benefit of the therapy. Moreover, we recognize that the study populations of 5 to 15 years have very active accommodation, and non-cycloplegic measure may not be extremely reliable. There are ongoing studies for the relationship between the myopia progression and the electrostimulation as its prevention [22-25]. These studies also suggest such physiotherapy increases blood circulation and metabolism in the surrounding tissues, and normalizes nervous systems in the area treated. This can be one of the proofs that myopia is a complex phenomenon.

Although two methods are still considered to have room for further development, our preliminary results showed improvement and prospect of new therapies since the treatment of myopia remains the prescription glasses or surgeries despite various efforts over the years.

Long-term effect of these therapies is one of the subjects for the future investigation. However, based on these results that both methods improved patients' vision, and 3D images improved distance vision more than near vision, and fogging improved near vision more, there may be different indications for these methods. 
Explaining these phenomena of vision improvements are not simple; however, similar to the fact that there are still no explanation to the difference between 95\% refraction $v s$ UCVA curve, we think this case demonstrate this gap [45]. As explained previously, the evolution of myopia and the mechanisms of its prevention still have many unknown issues, and the gap may not be the sufficient explanation.

Despite our general lack in knowledge, we believe our effort will give supports and encouragements to those who despair that juvenile myopia cannot be treated and only aggravate as the patient ages. In our clinical experience, we do observe the increase in visual acuity in myopic patients. The general belief that juvenile myopia being impossible to change is widespread not only among lay public, but also in the healthcare professionals as well. We are hopeful that we may be able to change this view in the future.

\section{CONFLICT OF INTEREST}

The authors confirm that this article content has no conflict of interest.

\section{ACKNOWLEDGEMENTS}

The authors thank the patients, legal guardians and the family of the patients, physicians, nurses, and administrators who participated in the study or helped us conducting the treatments and data collections.

Prototype of Sky Crystal OLYMPUS POWER 3D was supplied by Olympus Visual Communications. D'ACOMO and D-5000 Auto (World Optical Corporation, Kyoto, Japan) are properties of Denenchofu Eye Clinic.

\section{REFERENCE}

[1] Varghese RM, Sreenivas V, Puliyel JM, Varughese S. Refractive status at birth: its relation to newborn physical parameters at birth and gestational age. PLoS One 2009; 4(2): e4469.

[2] Kuo A, Sinatra RB, Donahue SP. Distribution of refractive error in healthy infants. J AAPOS 2003; 7(3): 174-7.

[3] Norton TT, Siegwart JT Jr. Animal models of emmetropization: matching axial length to the focal plane. J Am Optom Assoc 1995; 66(7): 405-14.

[4] Westall CA, Panton CM, Levin AV. Time courses for maturation of electroretinogram responses from infancy to adulthood. Doc Ophthalmol 1998-1999; 96(4): 355-79.

[5] Mutti DO. To emmetropize or not to emmetropize? The question for hyperopic development. Optom Vis Sci 2007; 84(2): 97-102.

[6] Atkinson J, Braddick O, Nardini M, Anker S. Infant hyperopia: detection, distribution, changes and correlates-outcomes from the cambridge infant screening programs. Optom Vis Sci 2007; 84(2): 84-96.

[7] Vitale S, Ellwein L, Cotch MF, Ferris FL, Sperduto R. Prevalence of refractive error in the United States, 1999-2004. Arch Ophthalmol 2008; 126(8): 1111-1119.

[8] Lawrence MS, Azar DT. Myopia and models and mechanisms of refractive error control. Ophthalmol Clin North Am 2002; 15(1): 127-33.

[9] Nallasamy S, Paluru PC, Devoto M, et al. Genetic linkage study of high-grade myopia in a Hutterite population from South Dakota. Mol Vis 2007; 13: 229-36.

[10] Lyhne N, Sjølie AK, Kyvik KO, Green A. The importance of genes and environment for ocular refraction and its determiners: a population based study among 20-45 year old twins. $\mathrm{Br} \mathrm{J}$ Ophthalmol 2001; 85: 1470-76.

[11] Taylor HR, Robin TA, Lansingh VC, Weih LM, Keeffe JE. A myopic shift in Australian Aboriginals: 1977-2000. Trans Am Ophthalmol Soc 2003; 101: 107-10.
[12] Edwards MH, Lam CS. The epidemiology of myopia in Hong Kong. Ann Acad Med Singapore 2004; 33(1): 34-8.

[13] He M, Zheng Y, Xiang F. Prevalence of myopia in urban and rural children in mainland China. Optom Vis Sci 2009; 86(1): 40-4.

[14] Saw SM, Gazzard G, Au Eong KG, Tan DT. Myopia: attempts to arrest progression. Br J Ophthalmol. 2002; 86(11): 1306-11.

[15] Allen PM, Radhakrishnan H, Rae S, et al. Aberration control and vision training as an effective means of improving accommodation in individuals with myopia. Invest Ophthalmol Vis Sci 2009; 50(11): 5120-9.

[16] Rawstron JA, Burley CD, Elder MJ. A systematic review of the applicability and efficacy of eye exercises. J Pediatr Ophthalmol Strabismus 2005; 42(2): 82-8.

[17] Liang CK, Ho TY, Li TC, et al. A combined therapy using stimulating auricular acupoints enhances lower-level atropine eyedrops when used for myopia control in school-aged children evaluated by a pilot randomized controlled clinical trial Complement Ther Med 2008; 16(6): 305-10.

[18] Chia A, Chua WH, Cheung YB, Wong WL, Lingham A, Fong A, Tan D. Atropine for the treatment of childhood myopia: safety and efficacy of $0.5 \%, 0.1 \%$, and $0.01 \%$ doses (Atropine for the Treatment of Myopia 2). Ophthalmology 2012; 119(2): 347-54.

[19] McBrien NA, Moghaddam HO, Reeder AP. Atropine reduces experimental myopia and eye enlargement via a nonaccommodative mechanism. Invest Ophthalmol Vis Sci 1993; 34(1): 205-15.

[20] Benavente-Perez A, Nour A, Troilo D. The effect of simultaneous negative and positive defocus on eye growth and development of refractive state in marmosets. Invest Ophthalmol Vis Sci 2012; 53(10): 6479-87.

[21] Gwiazda J. Treatment options for myopia. Optom Vis Sci 2009; 86(6): 624-8

[22] Ponomarchuk VS, Degtyarenko TV, Chaura AG, Lavrenko AN, Reshetnyak VB. Mechanisms responsible for the curative effect of phosphen-electrostimulation. Neurophysiology 1998; 30(6): 431-4.

[23] Degtyarenko TV, Ponomarchuk VS, Chaura AG. Dependence of the Curative Effects of Phosphene Electrostimulation on Its Frequency in Patients with Myopia. Neurophysiology 2002; 34(6): 462-7.

[24] Degtyarenko TV, Boichouk IM, Chaura AG. Changes in visual evoked potentials after phosphene electrostimulation in children suffering from myopia. Neurophysiology 2008; 40(3): 228-35.

[25] Walline JJ, Lindsley K, Vedula SS, Cotter SA, Mutti DO, Twelker JD. Interventions to slow progression of myopia in children. Cochrane Database Syst Rev 2011; 12:CD004916.

[26] Shiomi T, Uemoto K, Kojima T, et al. Simultaneous measurement of lens accommodation and convergence in natural and artificial 3D vision. J SID 2013. DOI\#:10.1002/jsid.156.

[27] Takada H, Matsuura Y, Miyao M. Effectiveness of new technology to compose stereoscopic movies, In "Depth Map and 3D Imaging Applications: Algorithms and Technologies", Malik AS, Choi TS, Nisar H, Eds. IGI Global, Hershey, PA, USA, Chapter 15, 2011. DOI\#:10.4018/978-1-61350-326-3.

[28] Sugiura A, Miyao M, Yamamoto T, Takada H. Effect of strategic accommodation training by wide stereoscopic movie presentation on myopic young people of visual acuity and asthenopia. Displays 2011; 32(4): 219-24.

[29] Takada M, Miyao M, Satoh M, Yoshikawa K, Matsuura Y, Takada $\mathrm{H}$. Effect of accommodation training on visual function of visual inspection workers and middle-aged people. J Sport Med Doping Stud 2012; 2(3). DOI\#: 10.4172/2161-0673.1000112.

[30] Ricci F, Cedrone C, Cerulli L. Standardized measurement of visual acuity. Ophthalmic Epidemiol 1998; 5(1): 41-53.

[31] Product information of D-5000 AUTO by World Optical Corporation, Kyoto, Japan. http://www.woc.co.jp/product/d5000.ht ml [Accessed: April 2, 2013].

[32] Holladay JT. Proper method for calculating average visual acuity. J Refract Surg 1997; 13(4): 388-91.

[33] GilmartingB. A Review of the role of sympathetic innervations of the ciliary muscle in ocular accommodation. Ophthalmic Physiol Opt 1986; 6(1): 23-37.

[34] Gilmartin B, Bullimore MA. Adaptation of tonic accomodation to sustained visual tasks in emmetropia and late-onset myopia. Optom Vis Sci 1991; 68(1): 22-6. 
[35] Gilmartin B, Hogan RE. The relationship between tonic accommodation and ciliary muscle innervation. Invest Ophthalmol Vis Sci 1985; 26(7): 1024-8.

[36] Charman H, Radhakrishnan H. Peripheral refraction and the development of refractive error: a review. Ophthal Physl Opt 2010; 30(4): 321-38.

[37] Phillips JR, McBrien NA. Pressure-induced changes in axial eye length of chick and tree shrew: significance of myofibroblasts in the sclera. Invest Ophthalmol Vis Sci 2004; 45(3): 758-63.

[38] García de la Cera E, Rodríguez G, Marcos S. Longitudinal changes of optical aberrations in normal and form-deprived myopic chick eyes. Vis Res 2006; 46(4): 579-89.

[39] Guggenheim JA, Northstone K, McMahon G, et al. Time outdoors and physical activity as predictors of incident myopia in childhood: a prospective cohort study. Invest Ophthalmol Vis Sci 2012; 53(6): 2856-65.
[40] Rose KA, Morgan IG, Ip J, Kifley A, Huynh S, Smith W, Mitchell P. Outdoor activity reduces the prevalence of myopia in children. Ophthalmology 2008; 115(8): 1279-85.

[41] Cottriall CL, McBrien NA. The M1 muscarinic antagonist pirenzepine reduces myopia and eye enlargement in the tree shrew. Invest Ophthalmol Vis Sci 1996; 37(7): 1368-79.

[42] Shih YF, Chen $\mathrm{CH}$, Chou AC, et al. Effects of different concentrations of atropine on controlling myopia in myopic children. J Ocul Pharmacol Ther 1999; 15(1): 85-90.

[43] Wesner MF, Miller RJ. Instrument myopia conceptions, misconceptions, and influencing factors. Doc Ophthalmol 1986 62(3): 281-308.

[44] Blade PJ, Candy TR. Validation of the PowerRefractor for measuring human infant refraction. Optom Vis Sci 2006; 83(6): 346-53.

[45] Holladay JT, Lynn MJ, Waring GO 3rd, et al. The relationship of visual acuity, refractive error, and pupil size after radial keratotomy. Arch Ophthalmol 1991; 109(1): 70-6.

(C) Ide et al.; Licensee Bentham Open.

This is an open access article licensed under the terms of the Creative Commons Attribution Non-Commercial License (http://creativecommons.org/licenses/by$\mathrm{nc} / 3.0 /$ ) which permits unrestricted, non-commercial use, distribution and reproduction in any medium, provided the work is properly cited. 